Amselle, Jean-Loup \& Sibeud, Emmanuelle, eds. -Maurice Delafosse. Entre orientalisme et ethnographie : l'itinéraire d'un africaniste (1870-1926). Paris, Maisonneuve \& Larose, 1998*

\title{
Sylvie KANDé
}

\section{OpenEdition}

\section{Journals}

Édition électronique

URL : http://journals.openedition.org/etudesafricaines/17

DOI : 10.4000/etudesafricaines.17

ISSN : 1777-5353

\section{Éditeur}

Éditions de l'EHESS

Édition imprimée

Date de publication : 1 janvier 2000

ISBN : 978-2-7132-1346-5

ISSN : 0008-0055

\section{Référence électronique}

Sylvie KANDé, « Amselle, Jean-Loup \& Sibeud, Emmanuelle, eds. -- Maurice Delafosse. Entre orientalisme et ethnographie : l'itinéraire d'un africaniste (1870-1926). Paris, Maisonneuve \& Larose, 1998* », Cahiers d'études africaines [En ligne], 157 | 2000, mis en ligne le 24 avril 2003, consulté le 21 septembre 2020. URL : http://journals.openedition.org/etudesafricaines/17 ; DOI : https://doi.org/10.4000/ etudesafricaines. 17

Ce document a été généré automatiquement le 21 septembre 2020

(c) Cahiers d'Études africaines 


\title{
Amselle, Jean-Loup \& Sibeud, Emmanuelle, eds. -- Maurice Delafosse. Entre orientalisme et ethnographie : l'itinéraire d'un africaniste (1870-1926). Paris, Maisonneuve \& Larose, 1998*
}

\author{
Sylvie KANDé
}

L'étude sur Maurice Delafosse que nous proposent Jean-Loup Amselle et Emmanuelle Sibeud reflète la mutation en cours dans l'appréciation des rapports entre individu et histoire : ni agent omnipotent ou charismatique, ni rouage mû par la machine économique, l'individu apparaît désormais comme le site intelligent, actif et contradictoire où/par lequel se fait une histoire en attente d'être organisée en récits. Ainsi remarque-t-on que Maurice Delafosse, par ses dates et ses pratiques, participe à la fois de la période vive de l'établissement de l'empire colonial et du long débat sur la classification des langues et des races, marqué par la fondation en 1925 de l'Institut d'ethnologie -- aboutissement partiel d'un projet de fusion de toutes les sociétés ethnographiques et moment d'institutionnalisation académique de l'ethnologie en France (cf. Filippo M. Zerilli : « Maurice Delafosse, entre science et action»). La compétence et le nombre des contributeurs aux récits raisonnés autour de Delafosse réunis dans ce recueil garantissent à la fois la densité de la réflexion collective et sa qualité dialogique.

Cet « itinéraire », qui est autant celui de Delafosse que des chercheurs qui se sont lancés sur sa trace, n'essaie donc pas -- contrairement à une biographie classique, telle que celle publiée par Louise Delafosse sous le titre Maurice Delafosse, le Berrichon conquis par l'Afrique ${ }^{1}$-- de couvrir dans l'ordre chronologique et de façon exhaustive les aspects principaux de l'homme, de sa vie, de son oeuvre. Il préfère saisir certains éléments des réalités de Delafosse, sans tentation hagiographique, sans recherche ni de cohérence 
absolue ni d'antithèses musclées. Particulièrement représentatif de la souplesse analytique de l'ensemble est l'article d'Emmanuelle Sibeud (« Les étapes d'un négrologue ») qui traite de la position charnière de Delafosse entre deux disciplines en continuité et rupture l'une par rapport à l'autre : l'ethnologie et les études africanistes. En vertu des logiques métisses de syncrétisme diachronique et synchronique de toutes les cultures, ou du principe de la Relation posé par Édouard Glissant selon lequel les histoires se présentent de façon rhizomatique plus que généalogique, cette étude s'attache à montrer en quoi Delafosse est un « ancêtre fondateur des études historiques sur l'Afrique » pour reprendre l'expression de l'historien Christophe Wondji dans la préface (p. 5). On se convainc, en lisant l'ouvrage, que Delafosse fut encore l'un des principaux inventeurs de l'Afrique, en raison de la part originale qu'il a prise à la constitution d'un discours sur l'Afrique qui puise, comme l'a établi ailleurs V. Y. Mudimbe, aux mythes et aux images de l'Antiquité.

Ce recueil est organisé autour de plusieurs équilibres paradoxaux qui caractérisent l'itinéraire de Delafosse -- le principal étant indiqué en sous-titre : entre orientalisme et ethnographie. Dans son article "L'Afrique par défaut ou l'oubli de l'orientalisme », Jean Schmitz souligne que c'est en orientaliste et arabisant de formation classique que Delafosse vient à l'Afrique; mais que, par ailleurs, il applique dans ses fonctions d'administrateur les principes participatoires de l'ethnologie de début de siècle, et ce, dans la tradition des travaux empiriques des Bureaux arabes. Ceux-ci visaient en effet à la constitution d'un savoir sur le pays conquis par fréquentation du terrain, pratique de la langue et prolongation des connaissances sur le passé antique régional, écrit en substance Jacques Frémeaux dans un texte intitulé « Des Bureaux arabes à Maurice Delafosse. Contribution à une étude de l'historiographie coloniale ». Ainsi, par ses collectes variées en tant que " savant de brousse ", par ses descriptions ethnographiques sur les Senufo entre autres (cf. Robert Launay, « A Question of Character : Delafosse among the Senufo »), par sa connaissance des langues locales et par son intérêt pour les textes arabes sur le Moyen âge africain, Delafosse a-t-il contribué à la réhabilitation de l'Afrique -- mieux, à la diffusion de la notion de civilisation africaine. Proche en cela de Frobenius, Delafosse rejoint aussi son collègue allemand sur l'importance de l'historicité des sociétés africaines dont tous deux tirent une double et paradoxale conclusion -- à savoir, la nécessité de rompre avec le paradigme évolutionniste, et celle d'établir la notion d'aires culturelles (cf. Benoît Hazard, « La construction de l'aire socioculturelle voltaïque dans l'oeuvre de Maurice Delafosse »), ce qui les mène tous deux à élaborer sur le concept d' « âme nègre » (cf. Clémens Zobel, « Essentialisme culturaliste et humanisme chez Leo Frobenius et Maurice Delafosse »).

Sauf à penser en termes de logiques métisses, paradoxale est encore la position de cet Occidental qui définit et défend une authenticité africaine sur l'hypothèse d'une UrKultur et d'une unité linguistique de l'Afrique, d'abord ; ensuite au moyen de l'étiquetage des ethnies (cf. Fabio Viti, « Dans un "chaos de races". Note sur Maurice Delafosse, administrateur des Anno-Mango »; Robert Nicolai, « Le songhay de HautSénégal-Niger à aujourd'hui : linéaments »); et par ce que J.-L. Amselle appelle " une véritable invention de l'animisme » (p. 127). Contre la double menace représentée par l'avancée de l'islam et sa capacité à organiser des empires, ainsi que par le projet colonial d'une politique d'assimilation des indigènes, Delafosse promeut un discours indigéniste, pan-nègre, voire panafricaniste, qui le place quelque part entre Blyden et Senghor. L'article de J.-L. Amselle, « Maurice Delafosse : un africaniste ambigu », 
indique bien en quoi les conclusions de Delafosse sur l'âme nègre, l'unité culturelle du continent, l'« ouverture » de l'Europe par opposition à la « fermeture » de l'Afrique ont pu servir de garantie scientifique à l'élaboration du mouvement de la Négritude et de référence à dépasser au dit « afrocentrisme » de Cheikh Anta Diop.

Le troisième grand paradoxe de l'oeuvre de Delafosse réside dans une oscillation constante entre discours d'élite et discours à l'usage du grand public ; entre discours scientifique et fiction littéraire. Tout en ayant fait oeuvre d'ethnologue et d'historien -oeuvre légitimée par « une combinaison de positions dans l'administration, dans l'enseignement, dans la presse coloniale, dans les sociétés savantes et les comités, dans le parti colonial lui-même » (Jean-Louis Triaud, « Haut-Sénégal-Niger, un modèle "positiviste" ? De la coutume à l'histoire : Maurice Delafosse et l'invention de l'histoire africaine », 226) --, Delafosse est aussi l'auteur d'une série d'articles intitulée «Broussard, ou les états d'âme d'un colonial ». Ils sont définis par Véronique Dimier, dans « Une analyse de l'administration coloniale signée Broussard », comme des textes de polémique politique, des fictions sensiblement autobiographiques, visant à expliquer le vécu de l'administrateur colonial à une audience métropolitaine, d'ailleurs plutôt réceptive, voire à recruter en son sein pour l'école coloniale. Bien plus : pour JeanHervé Jezequel (« Maurice Delafosse et l'émergence d'une littérature africaine à vocation scientifique »), Delafosse ferait partie de cette nouvelle génération de fonctionnaires coloniaux dont la rencontre avec les nouvelles élites africaines scolarisées, source de conflits politiques à maintes reprises (cf. Marc Michel, « Maurice Delafosse et l'invention d'une africanité nègre » sur le conflit emblématique entre Maurice Delafosse et Blaise Diagne), débouche aussi sur la production d'« études indigènes ", écrits à vocation scientifique, antérieurs et nécessaires à l'émergence de la littérature francophone.

L'ouvrage dirigé par J.-L. Amselle et E. Sibeud arrive à point nommé. Figure d'autorité scientifique et administrative pendant la phase active de sa carrière, Delafosse avait été jusqu'à présent un auteur négligé, quoiqu'une référence obligée, pour reprendre le titre de Marie-Albane de Suremain. Par ailleurs, ce recueil paraît à un moment où le mode de pensée binaire a perdu de son attrait heuristique, ouvrant un espace commun pour que l'Europe et l'Afrique puissent chacune apprécier -- au sens le plus neutre du terme -- au travers de la pensée et de l'action d'un Delafosse, les ambiguïtés de l'établissement d'un empire (du savoir) colonial. De ce travail extrêmement fouillé autour d'un personnage complexe -- orientaliste et ethnographe, chercheur et administrateur colonial, négrologue et anti-évolué, etc. -- qu'on peut donc imaginer comme un de ces sites intelligents, actifs et contradictoires où se dit l'histoire africaine et française, semble surgir un consensus : Delafosse reste celui qui a insisté « sur la nécessité d'une politique indigène qui serait aussi, à son avis, une politique plus humaniste, parce que fondée sur le véritable respect de l'homme africain » (Alice Conklin, «"On a semé la haine" : Maurice Delafosse et la politique du Gouvernement général en AOF, 1915-1936 », 65). On attend donc que le débat sur les multiples facettes de cette personnalité remarquable se poursuive avec des chercheurs d'universités africaines.

* Cet ouvrage fait l'objet d'un double compte rendu. 


\section{NOTES}

1. Paris, Société française d'histoire d'outre-mer, 1976. 